\title{
综合化学实验：5-澳-1-甲基芦竹碱衍生物的制备和表征
}

卢爱党 ${ }^{*}$, 李林, 史绍洋, 姜艳军 ${ }^{*}$

河北工业大学化工学院, 天津 300130

摘要: 设计了题为 “5-澳-1-甲基芦竹碱衍生物制备和表征” 的综合实验, 以5-溴吲哚为原料, 经Hofmann烷基化反应 和Mannich反应成功制备重要中间体5-澳-1-甲基芦竹碱, 然后与不同的酸反应制备系列5-澳-1-甲基芦竹碱衍生物。 结合化合物合成及表征, 既能提高学生的综合实验能力, 又能培养其对科学研究的兴趣和创新探索的精神。

关键词：综合实验；芦竹碱衍生物；Mannich反应；成盐反应

中图分类号: G64; O6

\section{A Comprehensive Chemical Experiment: Synthesis and Characterization of 5-Bromo-1-methylgramine Derivatives}

\author{
Aidang Lu ${ }^{*}$, Lin Li, Shaoyang Shi, Yanjun Jiang * \\ School of Chemical Engineering, Hebei University of Technology, Tianjin 300130, China.
}

\begin{abstract}
The synthesis and characterization of 5-bromo-1-methylgramine derivatives has been designed for comprehensive chemistry laboratory. 5-Bromo-1-methylgramine is synthesized by Hofmann alkylation, Mannich reaction, and salification reaction from 5-bromoindole. Then, a series of derivatives were obtained by reacting with different inorganic or organic acids. Combined with synthesis, characterization and analysis of the target compounds, the comprehensive experiment can not only improve the students' laboratory ability, but also stimulate their interest in scientific research and the spirit of innovation and exploration.
\end{abstract}

Key Words: Comprehensive experiment; Gramine derivatives; Mannich reaction; Salification reaction

实验教学在化学类本科生教学过程中的具有举足轻重的地位, 《普通高等学校本科专业类教育 质量国家标准》中也明确要求 “构建基础实验-综合性实验-研究性实验-多层次实验教学体系, 其中 综合性和研究性实验学时不低于总实验学时的 $20 \%{ }^{[1]}$ ”。为适应 “新时代” 教育的需求, 河北工业大 学应用化学专业打破传统的实验教学模式, 积极推进 “基础-综合-研究” 三层次实验教学体系, 开设 “创新性综合实验” 课程; 并鼓励教师结合科学前沿与研究课题发布实验题目供学生选择, 形成 “导 师制科研小团队” 进入科研实验室开展实验研究 ${ }^{[2]}$, 该课程连续开展了 4 学年, 学生反馈收获满满, 调查满意度高。本文以综合实验 “5-溴-1-甲基芦竹碱衍生物的制备和表征”为例进行详细介绍。

收稿: 2021-05-17; 录用: 2021-06-15; 网络发表: 2021-06-17

“通讯作者, Emails: luaidang@hebut.edu.cn (卢爱党); yanjunjiang@hebut.edu.cn (姜艳军)

基金资助: 河北省自然科学基金(B2020202028); 河北省重点研发计划项目(21326504D); 河北工业大学本科教育教学改革研究与实践项目 (202003022); 中国轻工业联合会教育工作分会课题(QGJY2020033) 


\section{1 实验简介}

\section{1 实验背景}

成盐是改善药物分子理化性质、提高其成药性的有效手段之一, 可在不改变其化学结构的基础 上通过成盐优化其理化性质、减少药物的不良反应、延长专利的保护期等 ${ }^{[3]}$, 如药物盐酸曲唑酮、磷 酸氯喹、甲氨基阿维菌素苯甲酸盐等。芦竹碱是小麦、大麦和芦竹等禾本植物在进化过程中产生的 具有化感作用的一种生物碱, 具有杀虫、杀菌、防污等活性 ${ }^{[4,5]}$ 。前期研究发现, 5-澳-1-甲基芦竹碱 对烟草花叶病毒具有优秀的抑制活性 ${ }^{[6]}$, 为了改善其水溶性、提高化合物的稳定性, 通过本实验的 开展制备系列成盐化合物进而进行深入研究。

\section{2 实验目的}

通过芦竹碱衍生物制备和表征，达到以下教学目的:

(1) 掌握几种重要的胺类化合物反应类型及其在官能团转化中的应用;

(2) 熟练运用柱层析法对化合物进行分离纯化并利用薄层层析色谱法检测反应的进程;

(3) 练习核磁共振数据处理软件 (MestReC)及 ChemDraw绘图软件的使用方法, 并能够解析核磁 共振波谱谱图。

\section{2 教学过程的组织与实施}

\section{1 实验教学计划}

本实验开设于应用化学专业本科生四年级秋季第6-9教学周(2学分, 40学时, 具体实验时间由指 导教师和学生灵活安排), 实验教学计划见表1, 实验内容可以由2-4人共同完成。

表1 实验教学计划

\begin{tabular}{clc}
\hline 实验进程 & \multicolumn{1}{c}{ 实验要求 } & 时间安排 \\
\hline 实验方案的设计 & 文献查阅及完成实验方案的设计 & 第6教学周 \\
实验内容的开展 & 完成5-溴-1-甲基芦竹碱衍生物的合成并进行表征 & 第7-8教学周 \\
数据整理与总结 & 整理实验数据, 撰写报告并进行总结汇报 & 第8-9教学周 \\
\hline
\end{tabular}

\section{2 实验方案设计及实验原理}

通过调研文献并结合5-澳-1-甲基芦竹碱结构特点, 确定实验方案如图1所示。实验原理为: 以5澳吲哚为原料, 首先对吲哚分子中氮原子进行甲基化, 然后与二甲胺和甲醛发生Mannich反应制备重 要中间体5-澳-1-甲基芦竹碱, 最后在不同无机酸和有机酸条件下制备得到5-澳-1-甲基芦竹碱成盐产 物。第一步, 5-溴吲哚与碘甲烷在氢氧化钾作用下发生亲核取代反应(霍夫曼(Hofmann)烷基化反应); 第二步, 在冰乙酸作用下, 5-澳-1-甲基芦竹碱吲哚与甲醛、二甲胺三组分发生Mannich反应得到5澳-1-甲基芦竹碱; 第三步, 由于氨基的氮原子上有一对孤对电子, 为质子接受体, 与不同的无机酸 或有机酸结合生成相应的盐, 反应式见图1。

\section{3 实验过程}

\subsection{1 试剂和仪器}

实验药品：5-溴吲哚、 $\mathrm{HN}\left(\mathrm{CH}_{3}\right)_{2}$ 水溶液(30\%)、 $\mathrm{HCHO}$ 水溶液(37\%)、冰乙酸、甲醇、 $N, N-$ 二甲基

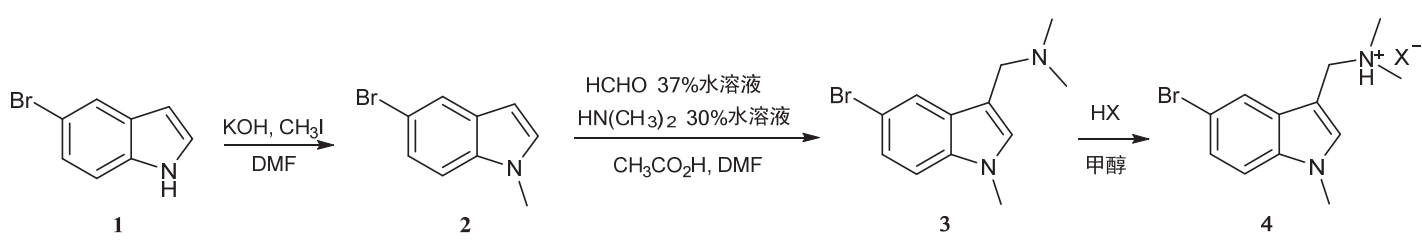

图1 5-溴-1-甲基芦竹碱衍生物的合成 
甲酰胺(DMF)、碘甲烷、二氯甲烷、乙醚、乙酸乙酯、苯甲酸、盐酸、氢澳酸、氢碘酸等, 所有试剂 均为分析纯, 购自天津市广达试剂。

实验仪器: DFY-5/80型低温恒温反应浴(巩义市京华仪器有限公司)、ZNCL-BS型磁力摚拌器、 Bruker Plus 400M型核磁共振波谱仪(德国Bruker)、RE-2000E型旋转蒸发仪(天津科诺仪器设备有限公 司)、ME303E型电子天平(梅特勒-托利多)、Waters 3100 Mass-Dector型质谱仪(Waters公司)、WFH-204B 型三用紫外分析仪(上海驰唐电子有限公司)、X-4数字显示显微熔点测定仪(巩义市予华仪器有限公 司)。

\subsection{2 实验操作}

\subsubsection{5-澳-1-甲基-1H-吲哚(2)的制备}

在 $0-5{ }^{\circ} \mathrm{C}$ 、磁力搅拌条件下, 将碘甲烷 $(6.4 \mathrm{~g}, 45 \mathrm{mmol})$ 滴加到 5 -溴吲哚 $(3.5 \mathrm{~g}, 30 \mathrm{mmol}) 、 \mathrm{KOH}$ $(6.7 \mathrm{~g}, 120 \mathrm{mmol}) 、 \mathrm{DMF}(50 \mathrm{~mL})$ 的混合溶液中, 滴加完毕后升至室温继续搅拌 $2 \mathrm{~h}$, 然后向反应体 系加入 $100 \mathrm{~mL}$ 水。水相用乙酸乙酯 $(100 \mathrm{~mL} \times 3)$ 进行萃取, 合并有机相并用水 $(100 \mathrm{~mL})$ 洗涤 3 次, 然 后用无水 $\mathrm{Na}_{2} \mathrm{SO}_{4}$ 干燥后真空脱溶, 残留液无需提纯得到淡黄色液体, 产率 $95 \%$, 可直接进行下一 步 $[7]$ 。

\subsubsection{5-溴-1-甲基芦竹碱(3)的制备}

在0 $-5{ }^{\circ} \mathrm{C}$ 、磁力搅拌下, 向 $150 \mathrm{~mL}$ 反应器加入DMF $(5 \mathrm{~mL}) 、 37 \% \mathrm{HCHO}$ 溶液 $(5 \mathrm{~mL}, 61.5 \mathrm{mmol})$ 、 $\mathrm{HN}\left(\mathrm{CH}_{3}\right)_{2} 30 \%$ 水溶液 $(5 \mathrm{~mL}, 33.5 \mathrm{mmol}), 1 \mathrm{~h}$ 后滴加 $2.86 \mathrm{~mL}$ 冰乙酸, 反应 $15 \mathrm{~min}$, 再加入 5 -溴-1-甲 基吲哚 $(3.2 \mathrm{~g}, 15.2 \mathrm{mmol})$ 反应 $1.5 \mathrm{~h}$ 后, 转移至室温反应 $6 \mathrm{~h}^{[6]}$ 。利用 TLC点板监测反应完毕后, 先加 入饱和食盐水, 再加入 $\mathrm{NaOH}(30 \%)$ 水溶液调节体系 $\mathrm{pH}=12-14$, 用 $\mathrm{CH}_{2} \mathrm{Cl}_{2}(30 \mathrm{~mL})$ 萃取三次, 有机 相依次用水和饱和食盐水洗涤, 合并有机相并用无水硫酸钠干燥, 过滤后真空脱溶。所得粗品经柱 层析提纯 (洗脱剂为 $V$ (石油醚) : $V$ (乙酸乙酯 $)=15: 1$ ) 得到淡黄色液体, 收率为 $85 \%$; ${ }^{1} \mathrm{H}$ NMR $(400$ $\left.\mathrm{MHz}, \mathrm{CDCl}_{3}\right) \delta 7.81(\mathrm{~d}, J=2.0 \mathrm{~Hz}, 1 \mathrm{H}, \mathrm{Ar}-\mathrm{H}), 7.29(\mathrm{dd}, J=2.0$ and $8.8 \mathrm{~Hz}, 1 \mathrm{H}, \mathrm{Ar}-\mathrm{H}), 7.15(\mathrm{~d}, J=8.4$ $\mathrm{Hz}, 1 \mathrm{H}, \mathrm{Ar}-\mathrm{H}), 6.98(\mathrm{~s}, 1 \mathrm{H}, \mathrm{Ar}-\mathrm{H}), 3.74\left(\mathrm{~s}, 3 \mathrm{H}, \mathrm{N}-\mathrm{CH}_{3}\right), 3.54\left(\mathrm{~s}, 2 \mathrm{H}, \mathrm{Ar}_{-} \mathrm{CH}_{2}\right), 2.26\left(\mathrm{~s}, 6 \mathrm{H}, \mathrm{N}-\left(\mathrm{CH}_{3}\right)_{2}\right)$; ${ }^{13} \mathrm{C} \mathrm{NMR}\left(100 \mathrm{MHz}, \mathrm{CDCl}_{3}\right) \delta 135.7,130.0,129.4,124.4,122.0,112.6,111.7,110.6,54.4,45.4$, 32.9; HRMS (ESI) calcd. for $\mathrm{C}_{12} \mathrm{H}_{16} \mathrm{BrN}_{2}(\mathrm{M}+\mathrm{H})^{+} 267.0491$, found 267.0496。

\subsubsection{5-溴-1-甲基芦竹碱盐(4)的制备}

将5-澳-1-甲基芦竹碱 $(0.17 \mathrm{~g}, 0.65 \mathrm{mmol})$ 溶于 $20 \mathrm{~mL}$ 甲醇中, 加入不同的酸 $(0.65 \mathrm{mmol})$, 加热回 流 $2 \mathrm{~h}$, 脱溶后, 用乙醚重结晶或柱层析 ( $V$ (二氯甲烷) : $V$ (甲醇 $)=20: 1$ )提纯得 5 -溴-1-甲基芦竹碱盐 $4 a-4 d$ 。

3· $\mathrm{HCl}(4 \mathrm{a})$ : 紫色固体, 收率 $96 \%$, 熔点 $220-221{ }^{\circ} \mathrm{C} ;{ }^{1} \mathrm{H}$ NMR $\left(400 \mathrm{MHz}, \mathrm{DMSO}-d_{6}\right) \delta 10.76(\mathrm{~s}, 1 \mathrm{H}$, $\left.\mathrm{N}^{+} \mathrm{H}\right), 8.12(\mathrm{~s}, 1 \mathrm{H}, \mathrm{Ar}-\mathrm{H}), 7.67(\mathrm{~s}, 1 \mathrm{H}, \mathrm{Ar}-\mathrm{H}), 7.49(\mathrm{~d}, J=8.7 \mathrm{~Hz}, 1 \mathrm{H}, \mathrm{Ar}-\mathrm{H}), 7.33(\mathrm{~d}, J=8.7 \mathrm{~Hz}, 1 \mathrm{H}$, $\mathrm{Ar}-\mathrm{H}), \quad 4.40\left(\mathrm{~d}, J=4.3 \mathrm{~Hz}, 2 \mathrm{H}, \mathrm{Ar}-\mathrm{CH}_{2}\right), 3.82\left(\mathrm{~s}, 3 \mathrm{H}, \mathrm{N}-\mathrm{CH}_{3}\right), 2.68\left(\mathrm{~d}, J=4.4 \mathrm{~Hz}, 6 \mathrm{H}, \mathrm{N}-\left(\mathrm{CH}_{3}\right)_{2}\right) ;{ }^{13} \mathrm{C}$ NMR $\left(100 \mathrm{MHz}, \mathrm{DMSO}-d_{6}\right) \delta 135.2,134.1,129.5,124.2,121.3,112.7,112.3,102.2,50.1,40.8$, 32.9 。

3· $\operatorname{HBr}(\mathbf{4 b})$ : 紫色固体, 收率 $93 \%$, 熔点 $212-214{ }^{\circ} \mathrm{C} ;{ }^{1} \mathrm{H}$ NMR $\left(400 \mathrm{MHz}, \mathrm{DMSO}-d_{6}\right) \delta 9.56(\mathrm{~s}, 1 \mathrm{H}$, $\left.\mathrm{N}^{+} \mathrm{H}\right), 8.11(\mathrm{~s}, 1 \mathrm{H}, \mathrm{Ar}-\mathrm{H}), 7.65(\mathrm{~s}, 1 \mathrm{H}, \mathrm{Ar}-\mathrm{H}), 7.45(\mathrm{~d}, J=8.7 \mathrm{~Hz}, 1 \mathrm{H}, \mathrm{Ar}-\mathrm{H}), 7.35$ (d, $J=8.7 \mathrm{~Hz}, 1 \mathrm{H}$, Ar-H), 4.45 (d, $\left.J=4.5 \mathrm{~Hz}, 2 \mathrm{H}, \mathrm{Ar}-\mathrm{CH}_{2}\right), 3.83\left(\mathrm{~s}, 3 \mathrm{H}, \mathrm{N}-\mathrm{CH}_{3}\right), 2.74\left(\mathrm{~d}, J=4.6 \mathrm{~Hz}, 6 \mathrm{H}, \mathrm{N}-\left(\mathrm{CH}_{3}\right)_{2}\right)$; ${ }^{13} \mathrm{C}$ NMR $\left(100 \mathrm{MHz}\right.$, DMSO- $\left.d_{6}\right) \delta 135.3,134.1,129.3,124.3,121.4,112.7,112.4,102.1,50.5,41.1$, 32.9 。

3.HI(4c): 紫色固体, 收率 $92 \%$, 熔点 $190-192{ }^{\circ} \mathrm{C} ;{ }^{1} \mathrm{H}$ NMR $\left(400 \mathrm{MHz}, \mathrm{DMSO}-d_{6}\right) \delta 9.36(\mathrm{~s}, 1 \mathrm{H}$, $\left.\mathrm{N}^{+} \mathrm{H}\right), 8.10(\mathrm{~s}, 1 \mathrm{H}, \mathrm{Ar}-\mathrm{H}), 7.65(\mathrm{~s}, 1 \mathrm{H}, \mathrm{Ar}-\mathrm{H}), 7.49(\mathrm{~d}, J=8.7 \mathrm{~Hz}, 1 \mathrm{H}, \mathrm{Ar}-\mathrm{H}), 7.34$ (d, J=7.5 Hz, 1H, Ar$\mathrm{H}), \quad 4.47\left(\mathrm{~d}, J=4.4 \mathrm{~Hz}, 2 \mathrm{H}, \mathrm{Ar}-\mathrm{CH}_{2}\right), \quad 3.84\left(\mathrm{~s}, 3 \mathrm{H}, \mathrm{N}-\mathrm{CH}_{3}\right), 2.78\left(\mathrm{~d}, J=4.5 \mathrm{~Hz}, 6 \mathrm{H}, \mathrm{N}-\left(\mathrm{CH}_{3}\right)_{2}\right)$; ${ }^{13} \mathrm{C} \mathrm{NMR}$ $\left(100 \mathrm{MHz}, \mathrm{DMSO}-d_{6}\right) \delta 135.3,134.0,129.2,124.4,121.4,112.7,112.4,102.1,50.7,41.3,33.0$ 。 
3·苯甲酸(4d): 黄色油状物, 收率为 $\left.95 \% ;{ }^{1} \mathrm{H} \mathrm{NMR} \mathrm{(400} \mathrm{MHz,} \mathrm{DMSO-} d_{6}\right) \delta 7.96(\mathrm{~d}, J=7.3 \mathrm{~Hz}, 2 \mathrm{H}$, Ar-H), 7.91 (d, $J=1.5 \mathrm{~Hz}, 1 \mathrm{H}, \mathrm{Ar}-\mathrm{H}), 7.55$ (t, $J=7.2 \mathrm{~Hz}, 1 \mathrm{H}, \mathrm{Ar}-\mathrm{H}), 7.40-7.47$ (m, 4H, Ar-H), 7.56 (dd, $J=8.7,1.7 \mathrm{~Hz}, 1 \mathrm{H}, \mathrm{Ar}-\mathrm{H}), 3.83\left(\mathrm{~s}, 2 \mathrm{H}, \mathrm{Ar}-\mathrm{CH}_{2}\right), 3.77\left(\mathrm{~s}, 3 \mathrm{H}, \mathrm{N}-\mathrm{CH}_{3}\right), 3.18\left(\mathrm{~s}, 1 \mathrm{H}, \mathrm{CO}_{2} \mathrm{H}\right), 2.35$ (s, $\left.6 \mathrm{H}, \mathrm{N}-\left(\mathrm{CH}_{3}\right)_{2}\right) ;{ }^{13} \mathrm{C} \mathrm{NMR}\left(100 \mathrm{MHz}, \mathrm{DMSO}-d_{6}\right) \delta 168.2,135.7,133.0,131.8,131.6,129.5,129.2$, $128.2,123.7,121.5,111.9,111.8,107.7,52.3,43.2,32.6$ 。

本阶段, 实验内容涉及理论知识点包括胺的碱性、霍夫曼(Hofmann)烷基化反应、Mannich反应、 胺与酸的成盐反应等, 涉及的实验操作包括萃取、真空脱溶、柱层析、加热回流、抽滤、TLC监测 等。学生不仅对相应知识点进行复习总结, 并掌握了化合物特别是特征官能团的性质; 而且综合运 用了有机化学实验教学中基本实验操作。

\section{4 数据整理与讨论}

\subsection{1 实验过程及思考}

实验过程中通过提出问题, 引导学生进行思考和自主学习。

(1) 胺的碱性: 实验方案设计阶段向学生提出问题, 5-溴-1-甲基芦竹碱分子结构中含有两个氮 原子, 与酸 1: 1 (摩尔比)反应时哪个氮原子与质子进行结合? 引导学生回顾学过的知识点分析两个 氮原子的碱性大小，并通过后续实验结果进行验证。

（2）霍夫曼Hofmann烷基化反应: 由于氮原子上有一对孤对电子, 容易与卤代烃发生亲核的取代 反应生成铵盐。提出问题: (1) 反应溶剂DMF在处理过程如何去除? (2) 不同卤代烃的反应活性大小 及如何提高胺类化合物中氮原子的亲核性?

(3) Mannich反应: 实验过程中用冰乙酸催化吲哚、甲酫、二甲胺3组分发生Mannich反应(反应机 理见图2), 反应条件的控制对反应收率的影响很大。除了实验中选择的实验方案外, 指导学生查阅 资料并总结还有哪些方法可以合成芦竹碱衍生物?
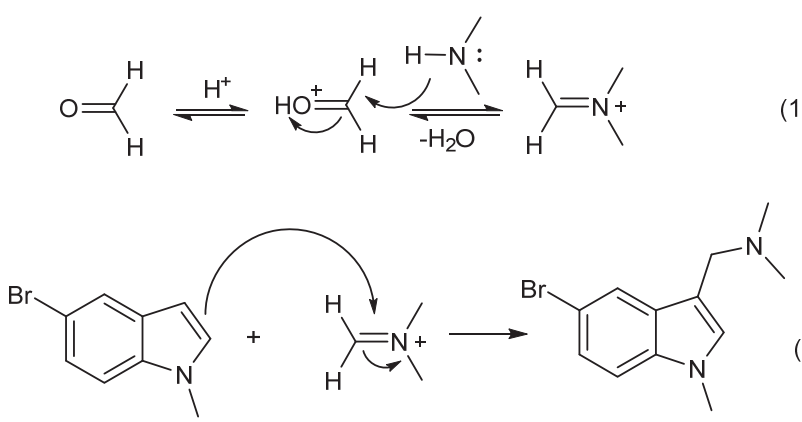

图2 Mannich反应机理

(4) 成盐反应: 回顾酸碱理论的知识, 5-溴-1-甲基芦竹碱与不同的酸反应是否能用酸碱质子理 论进行解释?

\subsubsection{5-溴-1-甲基芦竹碱的谱图解析}

根据5-澳-1-甲基芦竹碱的氢谱谱图(图3)所示, $\mathrm{N}\left(\mathrm{CH}_{3}\right)_{2}$ 处的 2 个甲基在 $\delta 2.26$ 处以单峰形式出现， $\mathrm{CH}_{2}$ 处 2 个氢原子在 $\delta 3.54$ 处以单峰形式出现。由于吲哚结构具有芳香性, 使得与氮原子相连的 $\mathrm{CH}_{3}$ 出 现的单峰向低场移动至 $\delta 3.74$ 。吲哚骨架的5位碳上溴原子电负性强, 使得邻近碳上质子受屏蔽降低, 质子位移向低场方向移动; 吲哚骨架的2位、4位、6位和7位的 $\mathrm{CH}$ 化学位移分别为6.98 (s)、7.81 (d, $J$ $=2.0 \mathrm{~Hz}) 、 7.29(\mathrm{dd}, J=2.0$ and $8.8 \mathrm{~Hz})$ 和 $7.15(\mathrm{~d}, J=8.4 \mathrm{~Hz})$, 4位 $\mathrm{CH}$ 由于间位碳上氢原子的影响发生 耦合出现双重峰, 6位 CH由于间位 CH以及邻位 $\mathrm{CH}$ 的共同影响发生耦合出现四重峰, 7位 CH由于邻位 $\mathrm{CH}$ 的影响发生耦合出现双重峰。高分辨质谱数据同样证明, 得到的化合物分子量与 5-澳-1-甲基芦竹 碱一致。 


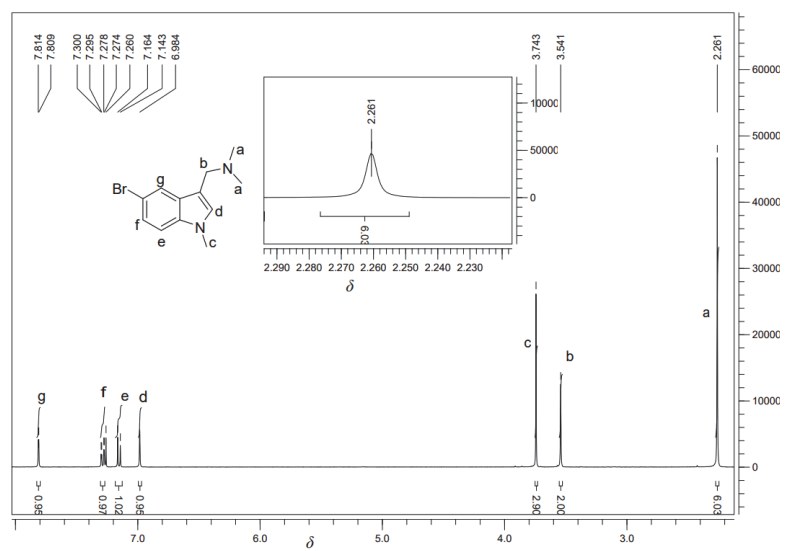

图3 5-溴-1-甲基芦竹碱的 ${ }^{1} \mathrm{H}$ NMR谱图

\subsection{3 化合物 4 的谱图解析}

对比 5-澳-1-甲基芦竹碱与无机酸 $\mathrm{HCl} 、 \mathrm{HBr}$ 及 $\mathrm{HI}$ 成盐后(4a-4c) 的 ${ }^{1} \mathrm{H} \mathrm{NMR}$ 谱图(4aa，见图4), $\mathrm{N}\left(\mathrm{CH}_{3}\right)_{2}$ 处的 2 个甲基在 $\delta 2.70$ 附近处均出现一个双重峰, 吲哚骨架中与氮原子相连的 $\mathrm{CH}_{3}$ 在 $\delta 3.80$ 附 近处仍出现一个单峰; 但 $\mathrm{CH}_{2}$ 处 2 个氢原子成盐后其化学位移向低场移动明显至在 $\delta 4.40$, 且为双重 峰。另外与无机酸 $\mathrm{HCl} 、 \mathrm{HBr}$ 及 $\mathrm{HI}$ 成盐后形成 $\mathrm{NH}^{+}$分别在 $\delta 10.76 、 9.56$ 和 9.36 新增一个单峰。实验结果 证明: $\mathrm{N}\left(\mathrm{CH}_{3}\right)_{2}$ 处氮原子比吲哚骨架中氮原子碱性强, 与酸反应后接受质子的氮原子为 $\mathrm{N}_{(}\left(\mathrm{CH}_{3}\right)_{2}$ 处氮 原子。脂肪胺由于烷基可提供电子使氮原子的电负性增强、碱性增强, 吲哚分子中苯环与吡咯环上 所有 $\pi$ 电子形成大 $\pi$ 体系, 使得氮原子结合质子的能力降低, 碱性变弱, 实验结果与前期理论分析相 一致。

对比苯甲酸盐 $4 \mathbf{d}$ (图4), $\mathrm{N}\left(\mathrm{CH}_{3}\right)_{2}$ 处的 2 个甲基在 $\delta 2.35$ 处仍出现一个单峰, 吲哚骨架中与氮原子 相连的 $\mathrm{CH}_{3}$ 在 $\delta 3.77$ 处仍出现一个单峰; 与苯甲酸成盐后 $\mathrm{CH}_{2}$ 处 2 个氢原子其化学位移向低场移动至 $\delta 3.83$ 处, 且仍为单峰形式出现; 大于 $\delta 9.0$ 处没有出峰, 而在 $\delta 3.18$ 出现一个单峰。由此确认, 与三 种无机酸(强酸)反应后氢离子与 5-澳-1-甲基芦竹碱形成共价键 $-\mathrm{N}^{+} \mathrm{H}$, 在氞代试剂DMSO中阴离子与 配阳离子之间为离子键; 但苯甲酸(弱酸)酸性较弱, 与5-澳-1-甲基芦竹碱只能以分子间作用力氢键 形式形成配合物, 此时酸上的氢并没有加到氮原子上成键, 因此对 $\mathrm{N}\left(\mathrm{CH}_{3}\right)_{2}$ 与和 $\mathrm{CH}_{2}$ 位移的影响就没 有强酸那么大, 类似现象已有文献报道 ${ }^{[8]}$ 。因此, 不能用酸碱质子理论解释 5 -溴-1-甲基芦竹碱与苯 甲酸的反应。

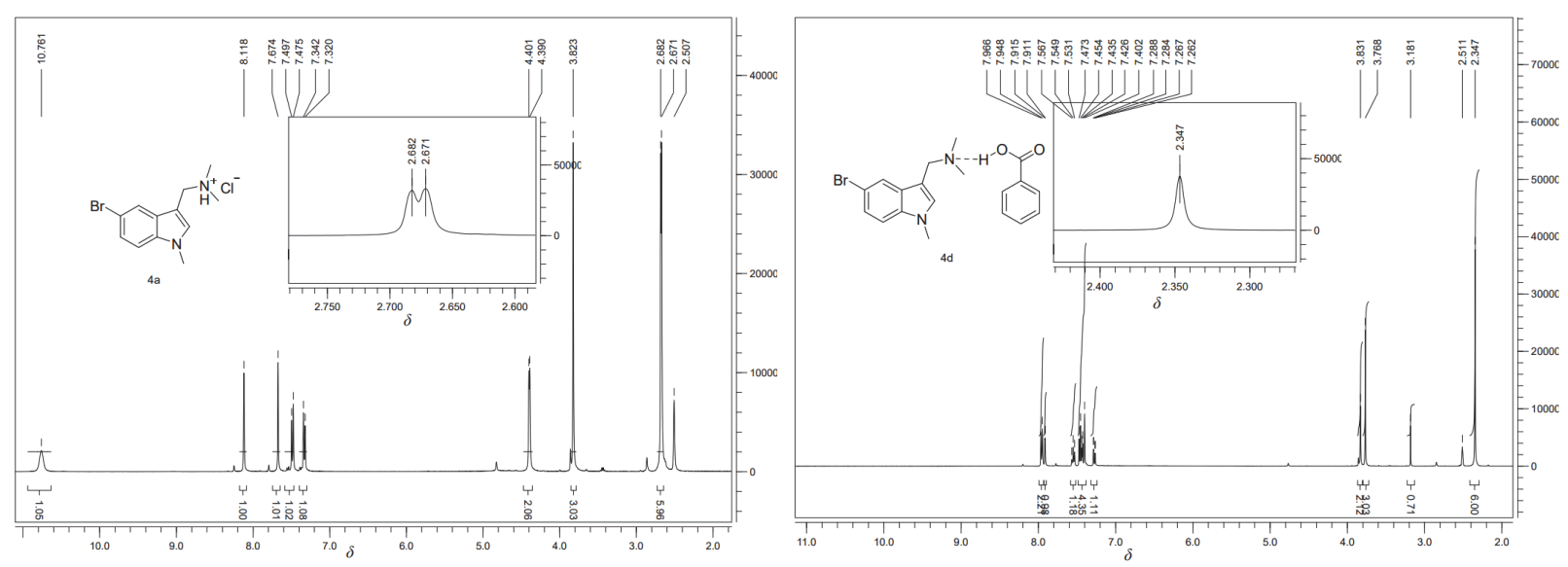

图4 化合物 $4 \mathrm{a}$ 和 $4 \mathrm{~d}$ 的 ${ }^{1} \mathrm{H}$ NMR谱图 
本阶段, 学生在完成5-澳-1-甲基芦竹碱衍生物制备和表征的基础上, 对实验数据进行整理、 分析及对比, 最后撰写实验报告。数据整理过程中不仅练习核磁共振数据处理软件 (MestReC)的使用 方法, 也提高了对化合物的 ${ }^{1} \mathrm{H} N \mathrm{NR} 、{ }^{13} \mathrm{C} N \mathrm{NR}$ 和HRMS谱图解析的能力。另外, 认真书写实验记录、 独立分析实验数据和翔实撰写实验报告的过程, 培养了学生严谨的科学态度, 调动了学生积极参与 科研的兴趣。

\subsection{4 实验安全注意事项}

实验过程中要着重强调用水、用电等实验室常规安全知识; 要求学生规范实验操作流程, 特别 如加热回流实验时要先通冷凝水后开启加热, 停止实验时要先关闭加热后停止通冷凝水, 使用旋转 蒸发仪前要认真学习仪器使用注意事项等。针对危险化学品的使用, 要求学生熟练使用化学品安全 技术说明书(MSDS)对化学品信息进行检索, 特别是具有强挥发性及刺激性气味的二甲胺水溶液、盐 酸、甲醛水溶液等, 具有低沸点的二氯甲烷、碘甲烷和乙醚等试剂。针对强挥发性和刺激性气味的 试剂注意好控制温度和尾气吸收等; 使用低沸点试剂时要注意好防护措施, 如在通风糜内进行实验。

\section{3 结语}

本实验设计了题为 “5-溴-1-甲基芦竹碱衍生物制备和表征” 的综合实验, 并应用于应用化学专 业创新性综合实验教学中。从反应原理、实验过程及结果分析三方面开展具有生物活性的5-溴-1-甲 基芦竹碱衍生物的制备, 提高学生对科学实验的熟悉程度, 使其参与并体验到科学研究具有的挑战 性和严谨性, 并为后续本科毕业论文做好铺垫。在实施效果上, 经过指导教师的引导以及与学生的 讨论后开始实施, 学生能结合不同章节知识顺利完成所有操作并撰写报告, 不仅夯实了他们的理论 知识和实验技能, 还能够训练学生调研、阅读及总结文献的能力, 并提高学生分析和解决问题的能 力, 有助于 “新工科” 专业人才的培养。开展5-溴-1-甲基芦竹碱衍生物制备的综合实验, 有助于学 生构建知识网络体系的同时, 强化课本理论知识与工程实践的融合, 培养学生科研兴趣, 并促进新 时代背景下高等院校专业人才的培养。

\section{参 考 文 献}

[1] 教育部高等学校教学指导委员会. 普通高等学校本科专业类教学质量国家标准(上). 北京: 高等教育出版社, 2018: 130.

[2] 卢爱党, 王铁男, 杨珊, 张东升. 化学教育(中英文), 2019, 40 (20), 40 .

[3] 沈芳, 苏颀, 周伟澄. 药物进展, 2012, 36 (4), 151.

[4] 许前会, 韦萍. 农药, 2004, 43 (2), 76 .

[5] 贝纳新, 高萍, 石承民, 田秀玲, 金亮. 沈阳农业大学学报, 2002, 33 (4), 309.

[6] Lu, A.; Tie, N.; Hui, H.; Wei, X.; Zhou, C.; Li, H.; Wang, Z.; Guo, J.; Ma, D.; Wang, Q. J. Agric. Food Chem. 2019, 67 (8), 2148.

[7] Dai, Z.; Li, S.; Li, Y.; Feng, L.; Ma, C. Tetrahedron 2019, 75 (13), 2012.

[8] 吴萌. 菲并吲哚里西啶生物碱的合成、结构改造、生物活性和构效关系研究[博士学位论文]. 天津: 南开大学, 2012. 